\title{
Shenqi Fuzheng Injection attenuates irradiation- induced brain injury in mice via inhibition of the NF-KB signaling pathway and microglial activation
}

\author{
Jian ZHANG ${ }^{1, \#}$, Fan TONG ${ }^{1, \#}$, Qian CAI ${ }^{1}$, Ling-juan $\mathrm{CHEN}^{1}$, Ji-hua DONG ${ }^{2}$, Gang WU ${ }^{1}$, Xiao-rong DONG ${ }^{1, \text { * }}$ \\ ${ }^{1}$ Cancer Center, ${ }^{2}$ Experimental Center, Union Hospital, Tongji Medical College, Huazhong University of Science and Technology, Wuhan \\ 430022, China
}

Aim: Radiation-induced brain injury (RIBI) is the most common and severe adverse effect induced by cranial radiation therapy (CRT). In the present study, we examined the effects of the traditional Chinese medicine Shenqi Fuzheng Injection (SFI) on RIBI in mice, and explored the underlying mechanisms.

Methods: C57BL/6J mice were subjected to a single dose of 20-Gy CRT. The mice were treated with SFI (20 mL-kg $\cdot \mathrm{d}^{-1}$, ip) for 4 weeks. Morris water maze test was used to assess the cognitive changes. Evans blue leakage and a horseradish peroxidase (HRP) assay were used to evaluate the integrity of the blood-brain barrier (BBB). The expression of inflammatory factors and microglial activation in brain tissues were detected using RT-PCR, Western blotting and immunofluorescence staining.

Results: CRT caused marked reductions in the body weight and life span of the mice, and significantly impaired their spatial learning. Furthermore, CRT significantly increased the BBB permeability, number of activated microglia, expression levels of TNF- $\alpha$ and IL-1 $\beta$, and the levels of phosphorylated p65 and PIDD-CC (the twice-cleaved fragment of p53-induced protein with a death domain) in the brain tissues. Four-week SFI treatment (administered for 2 weeks before and 2 weeks after CRT) not only significantly improved the physical status, survival, and spatial learning in CRT-treated mice, but also attenuated all the CRT-induced changes in the brain tissues. Four-week SFI pretreatment (administered for 4 weeks before CRT) was less effective.

Conclusion: Administration of SFI effectively attenuates irradiation-induced brain injury via inhibition of the NF-kB signaling pathway and microglial activation.

Keywords: Shenqi Fuzheng Injection; traditional Chinese medicine; cranial radiation therapy; radiation-induced brain injury; bloodbrain barrier; microglia; NF-kB

Acta Pharmacologica Sinica (2015) 36: 1288-1299; doi: 10.1038/aps.2015.69

\section{Introduction}

Cranial radiation therapy (CRT) is commonly used for the clinical treatment of primary or secondary brain tumors ${ }^{[1]}$. However, CRT causes many adverse side effects because it damages the adjacent brain tissues ${ }^{[2,3]}$. Radiation-induced brain injury (RIBI) remains a common and severe side effect, and approximately $50 \%$ of all survivors may develop lateonset radiation-induced injury of normal brain tissues ${ }^{[1,4-7]}$, which includes the progressive impairment of cognitive functions. Many pre-clinical studies have demonstrated that inflammatory responses tend to occur after CRT and may contribute to RIBI ${ }^{[8-10]}$, which suggests that neuro-inflammation is

\footnotetext{
\# These authors contributed equally to this work.

* To whom correspondence should be addressed.

E-mail tongfan.1986@163.com

Received 2015-01-17 Accepted 2015-06-15
}

implicated in RIBI ${ }^{[11-13]}$.

Microglia is considered a key mediator of neuro-inflammation $^{[14-16]}$. In the resting state, microglia exhibit a quiescent phenotype, which may play a role in the process of normal neurogenesis and suggests that microglia facilitate neurogenesis $^{[17]}$. Recent studies have shown that brain irradiation in vivo caused a conspicuous increase in activated microglia that was associated with a concomitant decrease in neurogenesis in the hippocampus and deficits in spatial memory retention ${ }^{[18]}$. These findings suggest that irradiation-induced microglial activation may play an important part in RIBI. In vitro studies have shown that irradiation could induce microglial activation, which, in turn, led to elevated expression of a variety of proinflammatory genes, including IL-1 $\beta$, IL-6, TNF- $\alpha$, and COX- $2^{[11]}$.

Moreover, the brain microenvironment is precisely regulated and protected for reliable neuronal signaling by specific barriers, including the blood-brain barrier $(\mathrm{BBB})^{[19]}$. The BBB 
controls the biochemical exchange between the blood and the brain parenchyma, with variable permeability ${ }^{[20]}$. The intact BBB plays an important role in maintaining the ionic homeostasis of brain, which is essential for the normal functions of central nervous system (CNS). When the BBB is disrupted, ionic movement, the excessive extravasation of proteins and biological response molecules, and the infiltration of inflammatory cells can all damage the brain tissue ${ }^{[21,22]}$.

Shenqi Fuzheng Injection (SFI) is extracted from a number of medicinal herbs, such as Radix Codonopsis (root of Codonopsis pilosula) and Radix Astragali (root of Astragalus), and was approved by the State Food and Drug Administration of the People's Republic of China in 1999. In patients with cancer, SFI reduces the side effects of chemotherapy ${ }^{[23]}$ and improves both immune function ${ }^{[24]}$ and the quality of life. A previous study revealed that SFI suppressed irradiation-induced inflammation principally by inhibiting TNF- $\alpha$ and IL-1 $\beta$ release and downregulating the expression of inflammatory factors in lung tissue ${ }^{[25]}$, but the exact mechanism remains elusive.

This study examined whether SFI could ameliorate RIBI in C57BL/6 mice and identify its mechanism by determining the permeability of $\mathrm{BBB}$, microglial activation, the release of inflammatory factors and the activity of the PIDD/NF- $\mathrm{BB}$ pathway in the brain after CRT.

\section{Materials and methods Animals}

Eight-week-old C57BL/6J male mice were obtained from Tongji Medical College, Wuhan, China. The mice weighing approximately $25 \mathrm{~g}$ were housed with $4-5$ animals per cage in laminar flow hoods and pathogen-free rooms to minimize the risk of infection. The animals were treated in strict accordance with the protocols approved by the Institutional Animal Care and Use Committee at Tongji Medical College, Wuhan, China. Efforts were made to minimize the number of animals used in the experiments and their suffering.

\section{Preparation of Shenqi Fuzheng for injection}

The SFI (Z19990065) has been identified and authenticated by the National Institute of Science Communication and Information Resources. According to previous research ${ }^{[26]}$, the injection was prepared using two medicinal herbs, Codonopsis pilosula (Campanulaceae) and Radix Astragali (Leguminosae), which were provided by Livzon Pharmaceutics Ltd (Zhuhai, China). Calycosin-7-O- $\beta$-glucoside, astragaloside IV and lobetyolin were the principal compounds in these two herbs ${ }^{[26]}$.

Because of the co-existence of multiple bioactive traditional Chinese medicinal products, high performance liquid chromatography (HPLC) analysis was performed to identify and evaluate the quality of the herbal preparations. The similarity between the test and control fingerprints (not less than 0.85) was set as the quality control index. The HPLC system in our study was used according to the manufacturer's protocol ${ }^{[26]}$. The Chromeleon 6.8 Chromatography Data System was used to calculate the peak areas. Water-acetonitrile composed the mobile phase (non-linear gradient, $40 \mathrm{~min}$ ), and a ZORBAX
Eclipse plus LC Column (C18, $3.0 \mathrm{~mm} \times 150 \mathrm{~mm}$, $3.5 \mathrm{~m}$, Agilent, Santa Clara, CA, USA) was used. All of the experiments were performed at $30^{\circ} \mathrm{C}$, the flow rate was $0.6 \mathrm{~mL} / \mathrm{min}$, and 266/288 nm UV absorbance detection was used for the analysis. Previous studies have reported that SFI was primarily composed of isoflavonoids, saponins, polysaccharides, pterocarpan glycosides, and alkaloids ${ }^{[25,27,28]}$. Therefore, calycosin7-O- $\beta$-glucoside, astragaloside IV and lobetyolin components were used as substance markers.

\section{Irradiation}

The animals were anesthetized with an intraperitoneal injection of Rompun at $0.6 \mathrm{mg} / 100 \mathrm{~g}$ body weight and ketamine at $0.5 \mathrm{mg} / 100 \mathrm{~g}$ in $0.9 \%$ saline. Then, the mice were given 20-Gy CRT, with the head centered in the exposure field. During the irradiation period, the eyes, neck, and body of the mice were protected with customized lead shielding. Because the mouse brain is more resistant to radiation injury than the human brain, a single dose of $20 \mathrm{~Gy}$ in the mouse approximates a clinically relevant dose in humans ${ }^{[29,30]}$. Irradiation was performed by employing a $6 \mathrm{MV}$ X-ray (SIEMENS, Germany). The dose was delivered to the mid-plane of the brain in one session through a posterior field. A plastic jig was used to fix the mice. The irradiation was administered with a beam energy of $6 \mathrm{MV}$ photons, a dose rate of $2.0 \mathrm{~Gy} / \mathrm{min}$, a sourcesurface distance (SSD) of $100 \mathrm{~cm}$, and an irradiation field size of $3 \mathrm{~cm} \times 30 \mathrm{~cm}$. Tissue-equivalent material (1-cm thick) was placed behind the head of each animal to achieve electronic equilibrium and thereby ensure uniform delivery. The mice were divided into five groups: (1) the B4RT group, which received SFI once daily for four consecutive weeks before 20-Gy CRT; (2) the B2RT group, in which SFI was administered for 2 weeks before and 2 weeks after the 20-Gy CRT; (3) the negative control RT-only group, in which the animals were given normal saline instead of SFI for 4 weeks before the whole brain was irradiated with a dose of 20-Gy CRT; (4) the SFI-only group, in which the animals were only given the intraperitoneal injection of SFI for 4 weeks; and (5) the normal control group, in which the animals did not receive any treatment. In each group, SFI $\left(20 \mathrm{~mL} \cdot \mathrm{kg}^{-1} \cdot \mathrm{d}^{-1}\right)$ was administered intraperitoneally (ip), as the earlier published reports ${ }^{[19,25]}$.

Three mice were chosen randomly for following examinations at each of the 8 time points: 3, 6, 24, 48, $72 \mathrm{~h}$, and 1, 2, 4 weeks after irradiation. The control animals at each time point were raised under identical conditions for the entire course of the experiment.

\section{Morris water maze}

To assess the cognitive changes after CRT, mice received either sham irradiation or a single dose of $20 \mathrm{~Gy}$. The Morris water maze test was conducted 6 weeks after CRT. The test was performed in a circular black pool (width: $127 \mathrm{~cm}$, height: $60 \mathrm{~cm}$ ) with a black platform. The pool was filled with milk diluted with water at room temperature $\left(250 \mathrm{mg} / \mathrm{L}, 21.0 \pm 1.0^{\circ} \mathrm{C}\right)$. The platform was set approximately $1.5 \mathrm{~cm}$ below the water surface.

The mice were placed into the water at 4 possible start loca- 
tions facing the wall of the pool, and a camera was simultaneously activated. Each mouse was allowed for up to $60 \mathrm{~s}$ to locate the platform. The trial was terminated when the mouse found the platform within the $60 \mathrm{~s}$. If a mouse failed to locate the platform after $60 \mathrm{~s}$, it was guided to locate the platform by a researcher and allowed to stay for 2-3 s. Each mouse was trained 3 times per day for $2 \mathrm{~d}$ to permit them to adapt to the pool environment (visible platform training) and was then tested 3 times per day for $5 \mathrm{~d}$ to find the hidden platform (hidden platform training). The latency (the time taken to find the platform in the water), distance, and swim speed were recorded by employing an automated video tracking software package (EthoVision 2.3.19, Noldus, Wageningen, The Netherland).

The pool was divided into four quadrants for further data analysis. The platform was moved to a different quadrant for each session in the visible training and was located in the center of the target quadrant, which was identical for the mice in the hidden platform training. The behavior of the mice was tracked using EthoVision 3.0 and analyzed for escape latency. In each trial, the average latency time for each mouse was calculated.

\section{Evans blue leakage and electron microscopic assessment of BBB integrity}

After the treatment with CRT and/or SFI, the functional integrity of the BBB was examined using Evans blue dye extravasation, according to a previously reported technique ${ }^{[9]}$, with some modifications, and the structural integrity of the BBB was assessed by electron microscopy. Forty-eight hours after CRT, $3 \mathrm{~mL} / \mathrm{kg}$ of $2 \%$ Evans blue dye (Sigma-Aldrich, St Louis, MO, USA) was injected via the femoral vein into the anesthetized mice. Several seconds after the injection, the conjunctivae and limbs turned blue, demonstrating a successful injection. Then, the brain was transcardially perfused with $200 \mathrm{~mL}$ of saline through the left ventricle until the perfusion fluid from the right atrium turned colorless. After decapitation, the brains were harvested, weighed, and then incubated in formamide solution at $50^{\circ} \mathrm{C}$ for $72 \mathrm{~h}$. The optical attenuation of the Evans blue formamide solution was spectrophotometrically determined at an excitation (emission) wavelength of 635 $\mathrm{nm}$, and the permeability of BBB was evaluated by the micrograms of Evans blue per milligram of brain tissue $(\mu \mathrm{g} / \mathrm{mg})$.

The ultrastructural changes were examined by a previously described horseradish peroxidase (HRP) assay at $48 \mathrm{~h}$ after $\mathrm{CRT}^{[31]}$, with minor modifications. After anesthesia, the animals received an intravenous injection of HRP (type II, Sigma Chemical Co, St Louis, MO, USA) through the catheter to acquire electron microscopic evidence of BBB permeability. Thirty minutes after HRP injection, the animals were transcardially perfused with saline $(50 \mathrm{~mL})$, followed by $0.1 \mathrm{~mol} / \mathrm{L}$ sodium phosphate buffer ( $\mathrm{pH} 7.4$ ) containing $4 \%$ paraformaldehyde and $2.5 \%$ glutaraldehyde. Subsequently, the brains were post-fixed with $2 \%$ osmium in phosphate buffer. The samples were dehydrated through a graded series of ethanol and embedded. Ultra-thin sections $(60 \mathrm{~nm})$ were examined with a transmission electron microscope (FEI Tecnai G2 20 TWIN, USA).

\section{General observations and body weight}

To observe the feeding and drinking behaviors, limb movement, and the local skin reaction to the irradiation, the mice were housed in the same conditions after the treatment with SFI or normal saline. Their body weight was recorded at each time point. All data were recorded and used as indicators of post-irradiation changes.

\section{RNA isolation and real-time RT-PCR}

RNA extraction, cDNA synthesis and real-time RT-PCR were performed as previously described ${ }^{[32]}$. Real-time PCR was used to analyze the cDNAs and was performed at $60^{\circ} \mathrm{C}$ to $95^{\circ} \mathrm{C}$ for 45 cycles on a Sequence Detection System (ABI Prism 7000, Applied Biosystems, Darmstadt, Germany) using the SYBR Green Reaction Master Mix (TaKaRa Biotechnology Co Ltd, Dalian, China), according to the manufacturer's instructions. The primers were: TNF-a sense: 5'-AGGCGGTGCCTATGTCTCA-3' and anti-sense: 5'-GAGGCCATTTGGGAACTTCT-3'; IL-1 $\beta$ sense: 5'-GAAATGCCACCTTTTGACAGTG-3' and anti-sense: 5' ${ }^{\prime}$-CTGGATGCTCTCATCAGGACA-3'; GAPDH sense: 5'-TCACCACCATGGAGAAGGC-3' and anti-sense: 5'-GCTAAGCAGTTGGTGGTGCA-3'. All primers were synthesized by Invitrogen (Groningen, Netherlands). GAPDH served as an internal control for every sample. The relative expression level of each target gene was calculated from the threshold cycle $(\mathrm{Ct})$ values against GAPDH gene, and the gene expression in the shamirradiated tissues was used as a control.

\section{Immunofluorescence staining}

The brain sections $(15 \mu \mathrm{m})$ were fixed in $4 \%$ paraformaldehyde for 3-4 $\mathrm{h}$ at room temperature and rinsed with PBS. After the sections were washed with PBS, the non-specific binding sites were blocked with $10 \%$ goat serum (GTX27481, GeneTex, USA) for $1 \mathrm{~h}$ at room temperature, and the samples were then incubated at $4{ }^{\circ} \mathrm{C}$ overnight with the following primary antibodies in 1\% goat serum: rabbit anti-mouse antibody Iba-1 (1:400, Wako, Japan), rat anti-mouse antibody F4/80 (1:50, AbD Serotec MCA497APCT, UK) and rabbit polyclonal antiTNF-a (1:50, ab9739, Abcam, Cambridge, MA USA). The sections were washed with PBS and incubated in the dark for $1 \mathrm{~h}$ with the following secondary antibodies in PBS: bovine Alexa Fluor 568 goat anti-rabbit IgG (H+L) (A11011, Invitrogen Life Technologies, Carlsbad, CA, USA) and Alexa Fluor 488 goat anti-rat IgG $(\mathrm{H}+\mathrm{L})$ (A11006, Invitrogen Life Technologies, Carlsbad, CA, USA). After the sections were washed with PBS three times for $5 \mathrm{~min}$, the nuclei were stained with DAPI (S36939, Invitrogen Life Technologies, Carlsbad, CA, USA) for $15 \mathrm{~min}$, and the sections were washed and mounted.

\section{Detection of apoptosis}

Apoptosis was detected in the hippocampal region by the terminal deoxynucleotidyl transferase dUTP nick-end labeling (TUNEL) assay, which was performed on deparaffinized tissue sections using the in situ Cell Death Detection Kit, Fluorescein (Roche Diagnostic GmbH, German), according to 
the manufacturer's protocol. Briefly, after deparaffinization and rehydration, the paraffin-embedded tissue sections were unmasked in citrate solution ( $\mathrm{pH}$ 6.0). Then, the sections were incubated with $50 \mu \mathrm{L}$ of the TUNEL reaction mixture $(5 \mu \mathrm{L}$ TdT enzyme solution, $45 \mu \mathrm{L}$ labeling solution) for $60 \mathrm{~min}$ at $37^{\circ} \mathrm{C}$ in a humidified atmosphere in the dark. Finally, the tissue sections were counterstained with DAPI and examined using a confocal microscope.

\section{Western blotting}

The proteins were extracted from the brain tissues using a protein extraction kit (Pierce Biotechnology Inc, IL, USA) according to the manufacturer's protocol. To determine the phosphorylation level of p65, the protein extracts were then separated on $15 \%$ sodium dodecyl sulfate polyacrylamide gels (SDS-PAGE). The proteins were transferred to a nitrocellulose membrane at $150 \mathrm{~mA}$ and then blocked with 5\% non-fat skim milk diluted with Tris-buffered saline containing $0.1 \%$ Tween 20 (TBST) for $1 \mathrm{~h}$ at room temperature. The mixture was incubated overnight with a primary antibody diluted with $2 \%$ bovine serum albumin in TBST at $4^{\circ} \mathrm{C}$. The primary antibodies were rabbit polyclonal IgG PIDD (1:200, Santa Cruz Biotechnology, Dallas, TX, USA) and rabbit anti-mouse phosphoNF-кB p65 (1:800, Cell Signaling Technology, Danvers, MA, USA). On the next day, the blots were incubated with the respective horseradish peroxidase-conjugated secondary
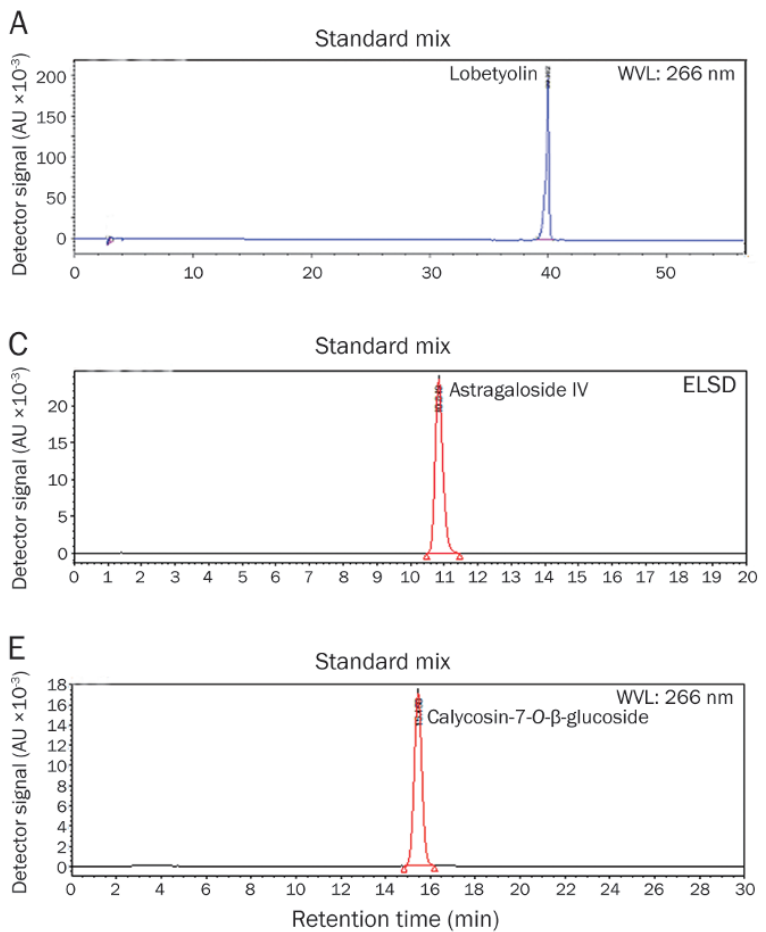

antibody (1:1000, Zhongshan Jinqiao Biotechnology Co, Ltd, Beijing, China), visualized using the enhanced chemiluminescence (ECL) detection system (BiYunTian Biotechnology Research Laboratory, Haimen, China), and then exposed to medical X-ray film. The intensity of the blots was quantified by employing a gel-image analyzer (JS380; Peiqing Science and Technology, Shanghai, China).

\section{Statistical analysis}

All of the quantitative data are expressed as the mean \pm SD. ANOVA was used for comparisons among more than two groups, followed by the appropriate post hoc test or ANOVA for repeated measurements. All tests were two-tailed. $P$ values less than 0.05 were considered statistically significant. The mouse survival curves were calculated with the Kaplan-Meier method and compared using the log-rank test with Statistical 6.0 software (Statsoft Statistica Enterprise, USA).

\section{Results}

\section{HPLC chromatograms of Shenqi Fuzheng Injection}

HPLC analysis showed that calycosin-7-O- $\beta$-glucoside, astragaloside IV and lobetyolin were the principle compounds of Radix Astragali and Radix Codonopsis using UV absorbance detection at $266 \mathrm{~nm}$ and ELS detection. Moreover, the HPLC analysis of SFI indicated that these three compounds were also principle components of SFI (Figure 1).
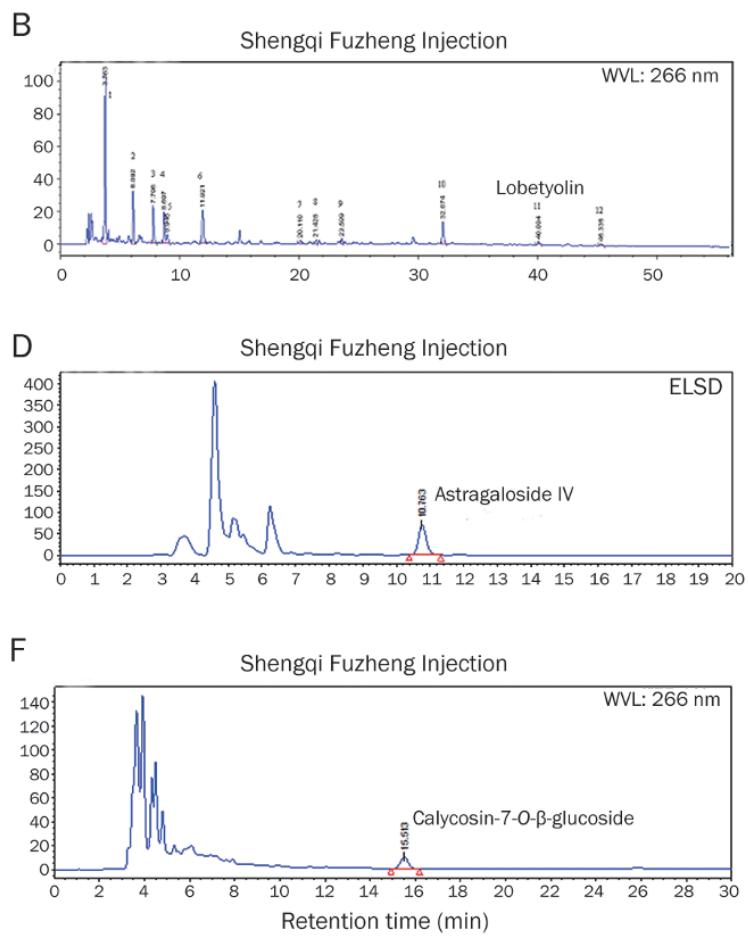

Figure 1. HPLC chromatograms of Shenqi Fuzheng Injection (SFI) using UV absorbance detection at 266 nm and ELS detection (ELSD). (A) The HPLC analysis of the standard mixture indicated that lobetyolin was detected by UV absorbance detection at $266 \mathrm{~nm}$. (B) The HPLC analysis of SFI indicated that lobetyolin was one of its compounds. (C) The HPLC analysis of the standard mixture showed astragaloside IV by UV absorbance detection at $266 \mathrm{~nm}$. (D) The HPLC analysis of SFI indicated that astragaloside IV was one of its compounds. (E) The HPLC analysis of the standard mixture showed calycosin-7-O- $\beta$-glucoside by UV absorbance detection at $266 \mathrm{~nm}$. (F) The HPLC analysis of SFI indicated that calycosin-7-O- $\beta$-glucoside was one of its compounds. 


\section{Effects of SFI on physical status and survival after CRT}

A total of 140 mice (28 mice per group) were used for the survival analysis. Our post-irradiation observations revealed that the mice in the RT, B4RT and B2RT groups showed mild local hair depilation and that their hair gradually turned white at the eighth week after CRT, but no evident difference was observed among these three groups (Figure 2A). Mice in the B4RT and B2RT groups were more active during the day, in terms of feeding and drinking, than were those in the RT-only group. No paralysis or convulsions were observed.

After CRT, the mice in all groups suffered from body weight loss, which was partially ameliorated by SFI. Other clinical indicators, such as general animal behavior, were also improved. Compared with the control, the body weight in

A
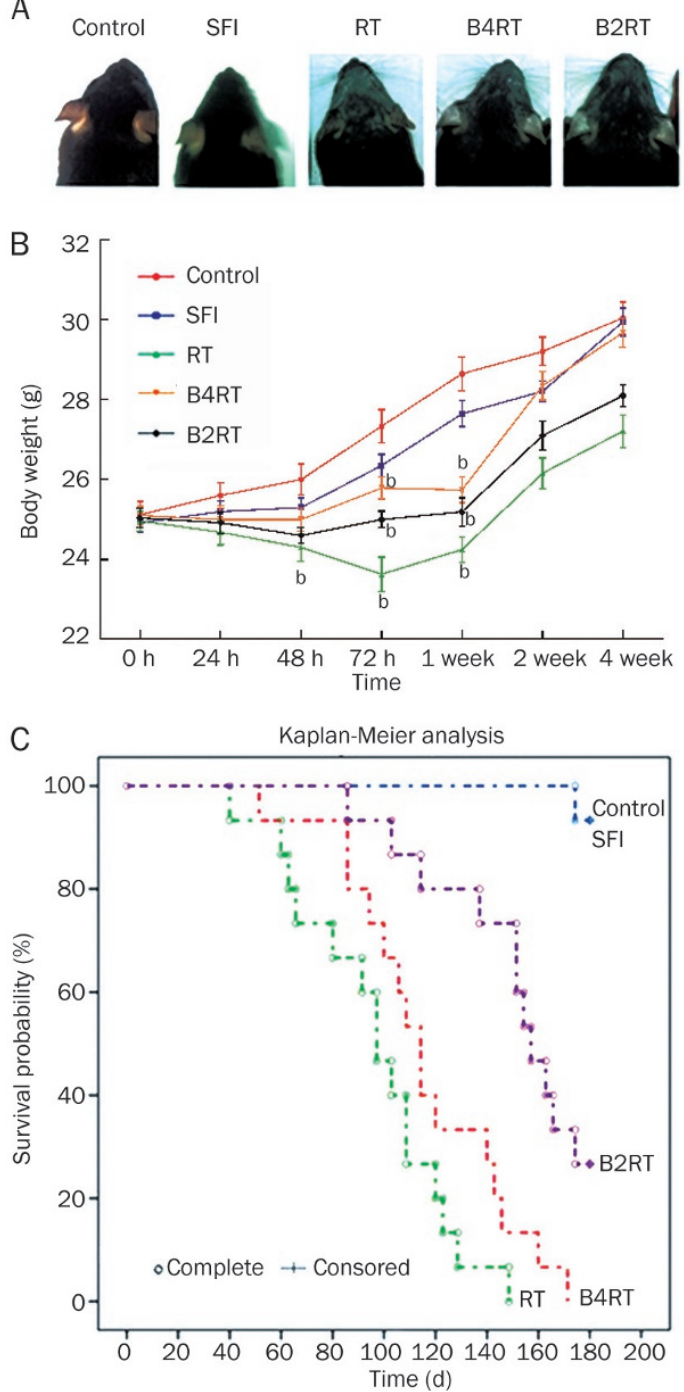

Figure 2. Effects of SFI on the physical status and survival of the mice after CRT. (A) The changes in the hair characteristics of each group. (B) The growth curves of body weight in each group. The data shown are the mean \pm SD. $n=5 .{ }^{b} P<0.05$ vs control. (C) Kaplan-Meier analysis of mouse survival in each group. RT, B4RT, B2RT vs control, $P<0.01$; B4RT vs RT, $P=0.042$; B2RT vs RT, $P=0.038$; B2RT vs B4RT, $P=0.059$. $n=28$. the RT-only group was significantly decreased at $48 \mathrm{~h}, 72 \mathrm{~h}$, and 1 week, and also in the B4RT and B2RT groups at $72 \mathrm{~h}$ and 1 week $(P<0.05)$. Two and four weeks after CRT, the body weight reversed to various degrees in the RT-only, B4RT, and B2RT groups ( $P>0.05$ vs control, Figure $2 \mathrm{~B})$.

Overall, 20-Gy CRT significantly reduced the life span of the mice (Figure 2C), with the median survival in the RTonly group lasting only for $95 \mathrm{~d}$. In contrast, the mice treated with SFI for 4 weeks had a median survival of $140 \mathrm{~d}$ (both SFI+RT groups vs RT-only group, $P<0.05$, log-rank test). The survival of the B2RT group was prolonged compared with the B4RT group, but the difference was not statistically significant $(P>0.05)$. The mice that received SFI only (SFI) had similar long survival as the sham-irradiated control animals. Together, the data showed that 4 weeks of SFI treatment improved survival after CRT, particularly in the B2RT group.

SFI alleviates irradiation-induced impairments in spatial learning, as shown by Morris water maze test

The mice were subjected to water maze trials with a visible platform ( 3 trials $/ \mathrm{d}$ for $2 \mathrm{~d}$ in a row). The irradiated mice reached the visible platform as rapidly as the control mice did in all 6 trials (data not shown), which indicated that their basic neurological functions (swimming, vision) were normal. For the hidden platform phase of the water maze test, all mice were trained for 3 sessions per day for 5 consecutive days. There was no significant difference in spatial learning between the RT groups and the control on d 1, 2 and 3 of the water maze test $(P>0.05)$. Interestingly, we observed that the latency was longer in the RT groups than in the control on $\mathrm{d} 4$ and $\mathrm{d} 5$ $(P<0.05$, Figure 3A). Compared with the RT-only group, the latency was reduced in the B4RT and B2RT groups on $\mathrm{d} 4$ and d 5 after treatment with SFI, but this difference was not significant $(P>0.05)$.

The total path length did not differ significantly between the RT-only group and the control on $\mathrm{d} 1,2$ and $3(P>0.05)$. However, the path length was longer in the RT-only group than in the control on $\mathrm{d} 4$ and $\mathrm{d} 5(P<0.05$, Figure $3 \mathrm{~B})$. Conversely, the path length was shortened in the B4RT and B2RT groups compared with the RT-only group, and the difference was significant between the B2RT and RT-only groups on $\mathrm{d} 5(P<0.05)$.

\section{SFI alleviats the irradiation-induced BBB damage}

Compared with the control, there was a significant increase in the amount of Evans blue dye in the three groups after irradiation (Figure 4A). The peak effect appeared at $48 \mathrm{~h}$ after CRT, and the Evans blue dye amount was $0.665 \pm 0.039 \mu \mathrm{g} / \mathrm{mg}$, $0.546 \pm 0.057 \mu \mathrm{g} / \mathrm{mg}$ and $0.460 \pm 0.030 \mu \mathrm{g} / \mathrm{mg}$, respectively, in the RT-only, B4RT and B2RT groups at $48 \mathrm{~h}$ after CRT. SFI significantly alleviated the irradiation-induced BBB permeability injury in the SFI-treated groups compared with the RTonly group at $48 \mathrm{~h}(P<0.01)$. In the control, no HRP reaction products were observed within the endothelial cells of the brain capillaries in the brain tissues of mice under ultrastructural observation (Figure 4B). CRT led to an obvious increase in the electron-dense HRP reaction products in the capillary 
A
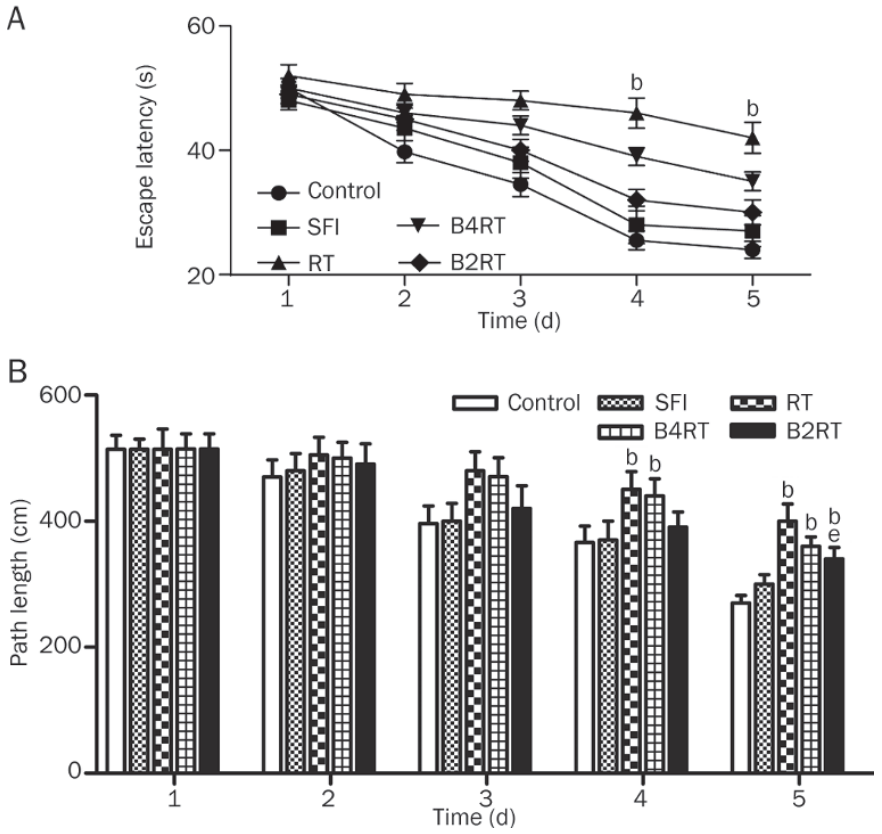

Figure 3. The effect of SFI on impaired spatial learning in five groups was observed using the Morris water maze test. (A) Escape latency in the training trials. (B) The path length to reach the platform. ${ }^{\mathrm{b}} P<0.05 \mathrm{vs}$ control group; ${ }^{e} P<0.05$ vs RT-only group. $n=5$.

endothelial cells, but in the SFI-treated groups, particularly in the B2RT group, the adjacent endothelial cells were intact and passage of HRP reaction products was reduced compared with the RT-only group.

Effect of SFI on the irradiation-induced increase in the mRNA expression of inflammatory factors

Figure 5 showed the real-time PCR results for the IL- $1 \beta$ and TNF-a mRNA levels in the brain tissues after CRT. After a single dose of $20 \mathrm{~Gy}$, a significant increase in TNF-a mRNA expression was detected in the brain tissues as early as $3 \mathrm{~h}$ after CRT. Then, the TNF- $a$ mRNA level returned to the baseline level at 24, 48, $72 \mathrm{~h}$ and 1 week after the irradiation. TNF-a mRNA expression was significantly increased and exhibited a second peak at 4 weeks after the irradiation compared with the control $(P<0.01)$. However, SFI significantly decreased TNF- $a$ mRNA expression at all of the time points, particularly in the B2RT group, compared with the RT-only group at $3 \mathrm{~h}$ and 4 weeks after irradiation $(P<0.01$, Figure $5 \mathrm{~A})$.

Low IL-1 $\beta$ mRNA expression level was observed in the brain tissues of the control animals. After irradiation with a single dose of $20 \mathrm{~Gy}$, irradiation-induced IL- $1 \beta$ mRNA expression was significantly increased in the brain tissues at 3,6 , and $24 \mathrm{~h}$ after the irradiation compared with the control $(P<0.01)$. Then, the irradiation-induced IL-1 $\beta$ mRNA expression returned to the baseline level at 48 and $72 \mathrm{~h}$ after irradiation. The irradiation-induced IL-1 $\beta$ mRNA expression was significantly elevated at 1,2 , and 4 weeks after irradiation compared with the control $(P<0.01)$. The IL-1 $\beta$ mRNA expression

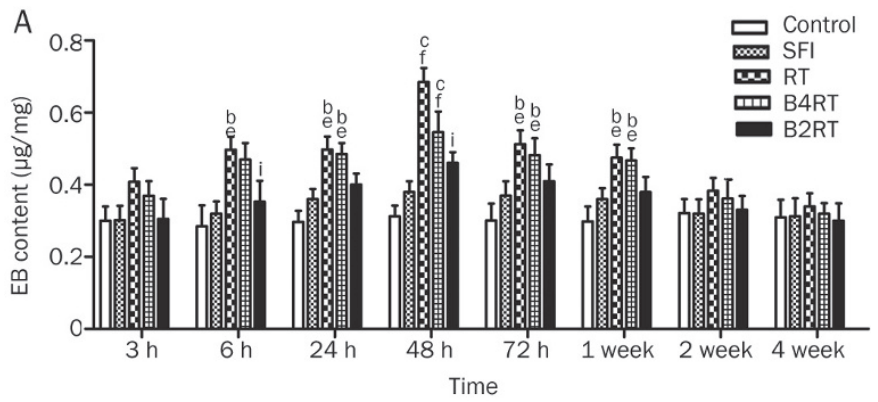

B

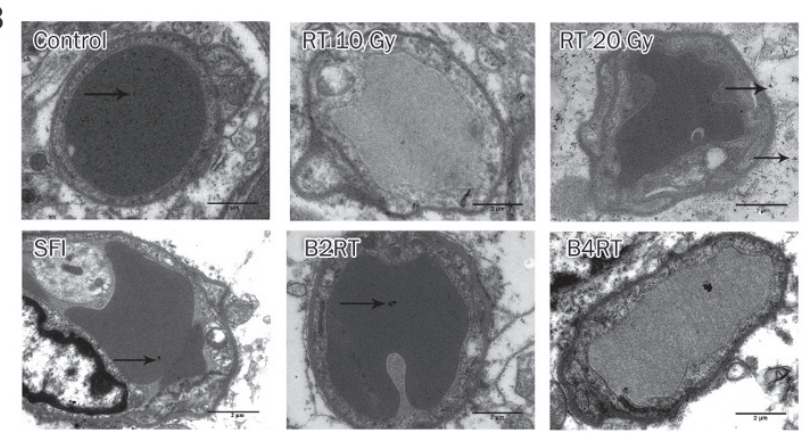

Figure 4. SFI alleviated irradiation-induced BBB damage. (A) Evans blue (EB) leakage assessment of BBB integrity. ${ }^{\mathrm{b}} P<0.05,{ }^{\mathrm{C}} P<0.01$ vs control; ${ }^{\mathrm{e}} P<0.05,{ }^{\mathrm{f}} P<0.01$ vs only SFI groups; ' $P<0.01$ vs RT group. (B) Electron microscopic assessment of horseradish peroxidase (HRP) permeability at $48 \mathrm{~h}$. The arrows were used to indicate the HRP reaction products. Scale bar $=2 \mu \mathrm{m}$.

A

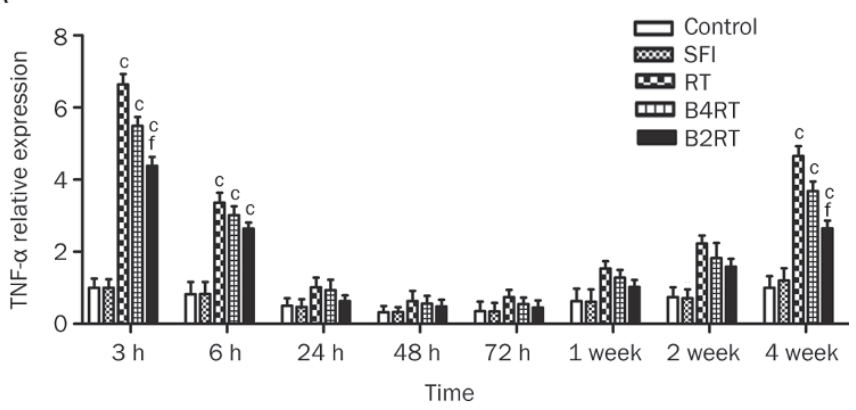

B

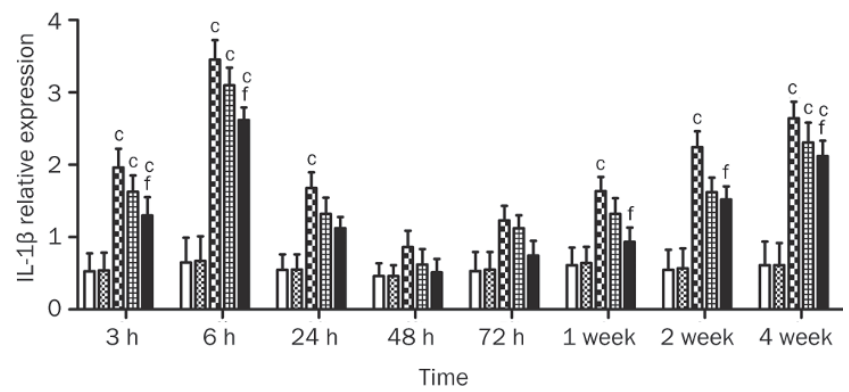

Figure 5. Effect of SFI on the TNF- $\alpha$ and IL-1 $\beta$ mRNA expression after CRT. (A) Real-time PCR for the TNF- $\alpha$ mRNA level. (B) Real-time PCR for the IL-1 $\beta$ mRNA level. ${ }^{c} P<0.01$ vs control; ${ }^{f} P<0.01$ vs RT-only group. 
reached a maximal level at 4 weeks post-irradiation. Then, we examined the effect of SFI on the post-irradiation expression of IL- $1 \beta$ and found that the irradiation-induced expression of IL-1 $\beta$ was decreased after treatment with SFI at all of the time points, particularly in the B2RT group at $3 \mathrm{~h}, 6 \mathrm{~h}, 1,2$ and 4 weeks, compared with the RT-only group $(P<0.01$, Figure $5 \mathrm{~B})$.

\section{Up-regulation of PIDD expression and inhibition of NF-KB activation in mice with irradiation-induced brain injury}

Western blotting revealed that the expression of phosphorylated p65 (p-p65)was obviously low in the control (Figure 6A and $6 \mathrm{~B})$. However, the expression was evidently increased after 20-Gy CRT. SFI inhibited the expression of p-p65 in the B4RT and B2RT groups to different extents. These findings suggested that SFI could inhibit NF-kB activation. The inhibitory effect of SFI on NF-kB pathway activation was more conspicuous in the B2RT group than in the B4RT group $(P<0.05$, Figure 6A and 6B).

We then examined the change of PIDD in brain tissues after irradiation (Figure 6C and 6D). Western blotting showed three protein segments, PIDD-FL (full-length p53-induced protein segments with a death domain), PIDD-C, and PIDD-CC, in the brain tissues, with molecular weights of 100,51 , and $37 \mathrm{kDa}$, respectively. Only a marginal expression of PIDD-FL was detected in the control and RT groups at all of the time points (Figure 6D). An obvious increase in PIDD-C was detected at 3 and 72 h, 2, and 4 weeks after CRT (Figure 6D). PIDD-CC was significantly increased after CRT compared with the control, and it reached a peak at the $3 \mathrm{~h}$ after CRT (Figure 6D). Moreover, the expression of PIDD-C and PIDD$\mathrm{CC}$ was reduced after SFI, and the differences were significant between the RT group and the B2RT group.

\section{Histological and immunohistochemical examination of brain tissues}

The total number of microglia (Iba-1-positive cells) in the hippocampus was counted to assess the changes in the microglial population after whole-brain irradiation (Figure 7A). Ionized calcium-binding adaptor molecule 1 (Iba-1) is located in the cytosol of all microglia and infiltrating monocytes irrespective of activation status, although its expression is increased after activation. Our study showed that $3 \mathrm{~h}$ after 20-Gy CRT, the number of Iba-1-positive microglia in 1- $\mathrm{mm}^{2} \mathrm{CA} 1$ region of hippocampus was significantly reduced compared with the control $(P<0.01$, Figure $7 \mathrm{~B})$. In the B2RT and B4RT groups, there was also a decrease in the Iba-1-positive microglia. Compared with the RT-only group, SFI increased the number of CRT-reduced microglia, particularly in the B2RT group $(P<0.01)$.

The expression of F4/80, which is specific for activated microglia, was significantly elevated after 20-Gy irradiation, which suggests that the microglia were activated by the irradiation. Staining with anti-F4/80 antibody showed that the number of activated microglia (positive for both Iba-1 and F4/80) was significantly increased at $3 \mathrm{~h}$ after CRT $(P<0.05$; Figure $7 \mathrm{~B})$. The results indicated that SFI could decrease the number of activated microglia at $3 \mathrm{~h}$ after CRT in the B4RT and B2RT groups $(P>0.05)$.

We then analyzed the expression of TNF-a and F4/80 by immunofluorescence staining in the hippocampus at $24 \mathrm{~h}$ after CRT and found that SFI inhibited TNF- $\alpha$ and F4/80 expression in the hippocampus, particularly in the B2RT group (Figure 8A).

Additionally, apoptosis was observed by TUNEL analysis (Figure 8B). In the sham-irradiated and SFI-only treatment groups, the apoptotic cells in the hippocampus were nearly absent. After $24 \mathrm{~h}$ of CRT, the number of TUNEL positive cells was increased, and SFI treatment could decrease the number of apoptotic cells after CRT.

\section{Discussion}

Most patients who survive for more than 6 months after CRT tend to develop irradiation-induced cognitive changes, called RIBI, which are predominantly manifested as deficits in learning, memory, and spatial information processing and are hippocampal-dependent functions ${ }^{[33]}$. A recent study suggested that neuro-inflammation, which is accompanied by the activation of microglial cells and the release of pro-inflammatory cytokines, played key roles in brain injury ${ }^{[22]}$.

SFI is extracted from a number of medicinal herbs, such as Radix Codonopsis (root of Codonopsis pilosula) and Radix Astragali (root of Astragalus), and was approved by the State Food and Drug Administration of the People's Republic of China in $1999^{[34,35]}$. SFI is a compound preparation that primarily contains calycosin-7-O- $\beta$-glucoside, astragaloside IV and lobetyolin. There is no literature suggesting which component of SFI plays a major role in microglial activation, and the mechanism remains unknown. However, many studies have shown that the main components of SFI have antiinflammatory effects. It was found that the extracts of Chinese medicinal herbs, such as Codonopsis pilosula, stimulate IL-1 ${ }^{[36]}$. Additionally, it was found that Astragalus mongholicus polysaccharides could inhibit the LPS-induced production of TNF-a and IL-8 mRNAs, possibly by suppressing the p38 signaling pathway ${ }^{[37]}$. Because microglial activation is the main source of neuro-inflammation ${ }^{[38]}$, the microglia may be the target of SFI that contributes to the anti-inflammatory effect.

Additionally, there were previous reports regarding the two main components (lancemaside A and Myelophil) and their effects on the nervous system. It was reported that lancemaside A, the main component of Codonopsis, could significantly reverse scopolamine-induced memory and learning deficits by increasing the expression of brain-derived neurotrophic factor (BDNF) and phosphorylated cAMP response element binding protein $^{[39]}$. Another article indicated that Myelophil, a combination of extracts taken from Astragali radix and Salviae miltiorrhizae radix, had anti-amnesic properties in a mouse model of memory impairment by ameliorating the expression of both ERK and muscarinic acetylcholine receptor 1 (mAChR1) ${ }^{[40]}$.

Our study demonstrated that intraperitoneal injection of SFI after CRT significantly ameliorated learning and memory deficits, decreased BBB permeability, suppressed inflammatory 
A

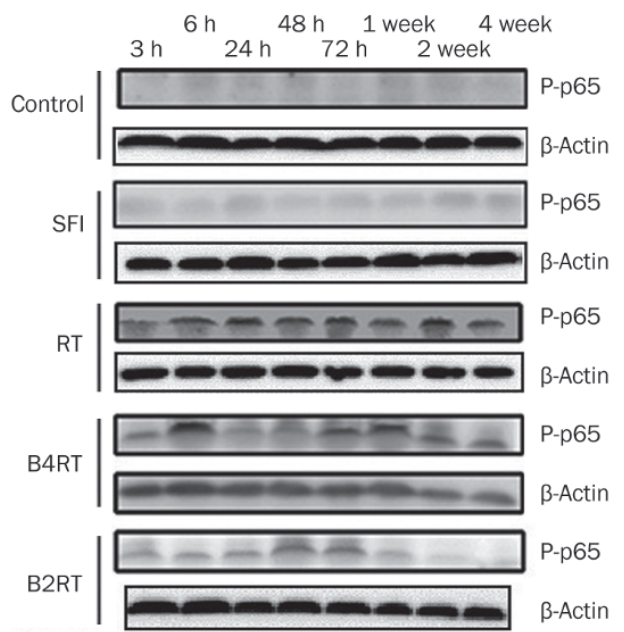

C
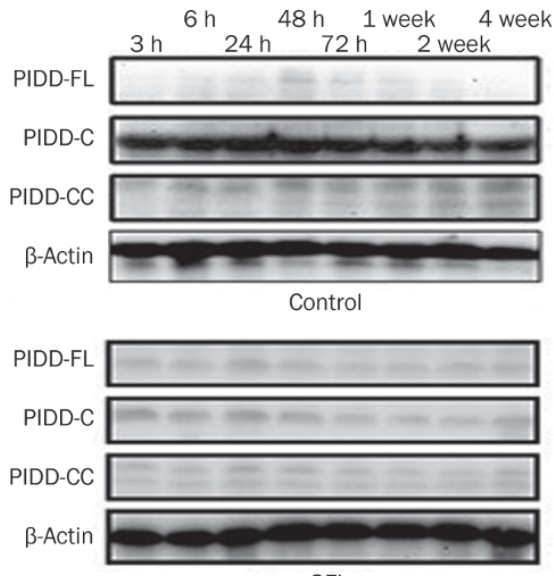

$\mathrm{SFI}$

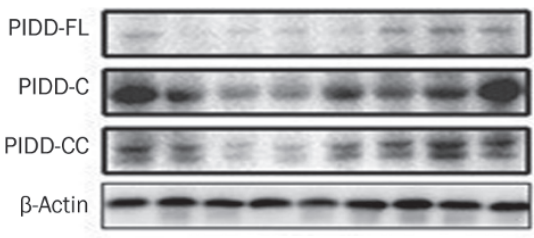

RT

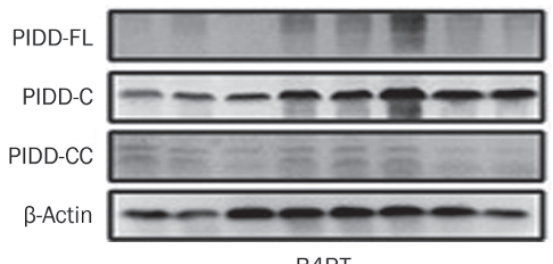

B4RT

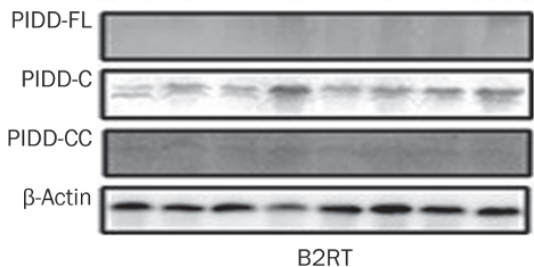

B
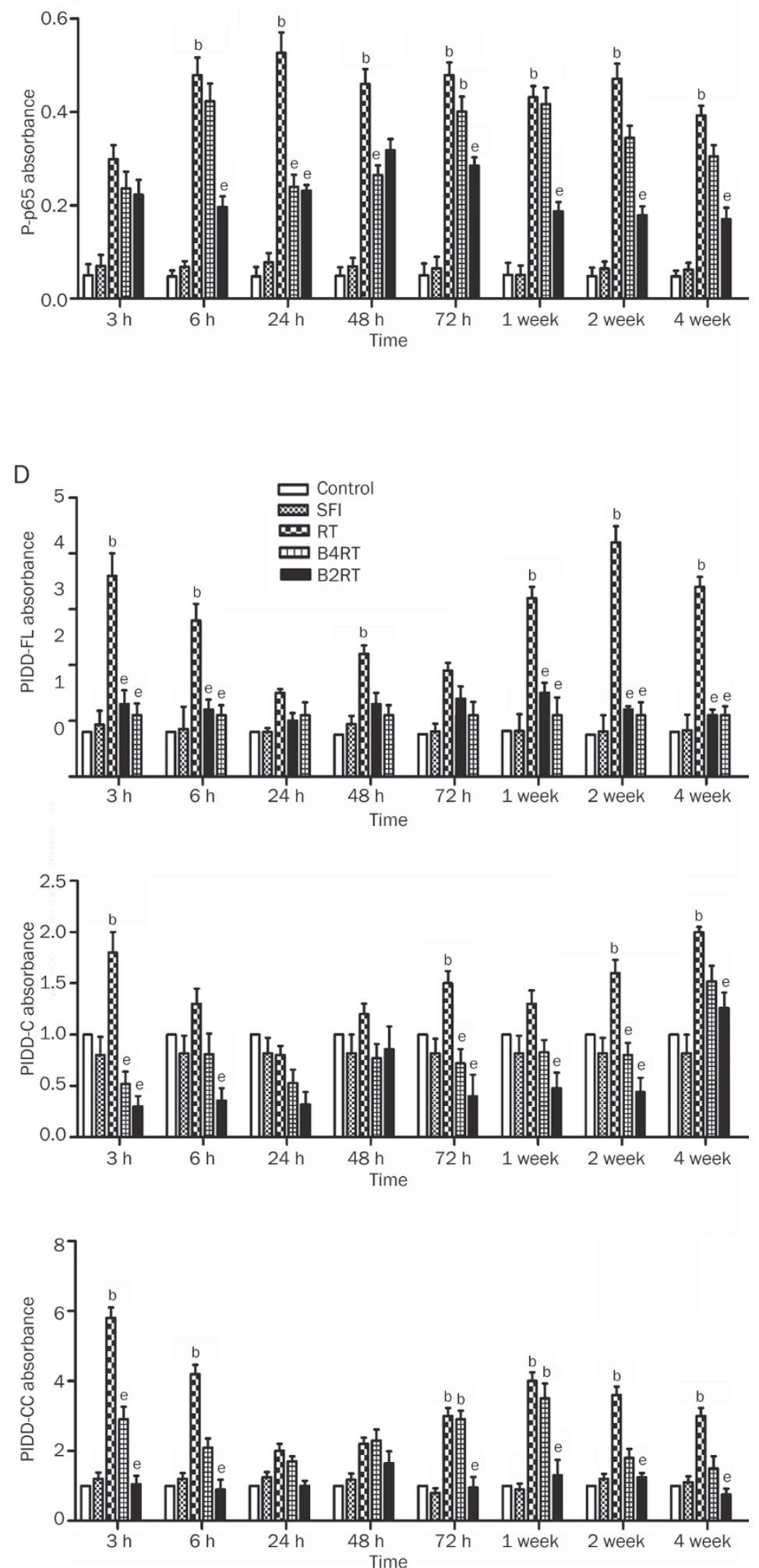

Figure 6. Up-regulation of PIDD expression and inhibition of NF-KB activation in mice with irradiation-induced brain injury. (A) Western blotting analysis for phosphorylated p65 (p-p65). (B) Relative quantity analysis for Western blotting of $p-p 65$. ${ }^{b} P<0.05$ vs control; ${ }^{e} P<0.05$ vs RT-only group. (C) Western blotting analysis for PIDD-FL, PDD-C and PIDD-CC. (D) Relative quantity analysis for Western blotting of PIDD-FL, PDD-C and PIDD-CC. ${ }^{\mathrm{b}} \mathrm{P}<0.05$ vs ontrol; ${ }^{e} P<0.05$ vs RT-only group. 
A

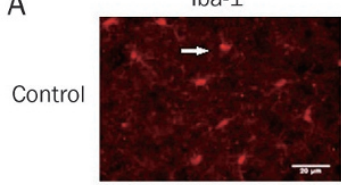

$\mathrm{SFI}$

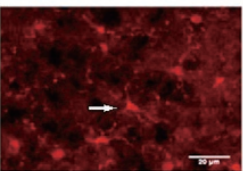

RT

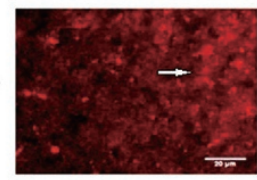

B2RT

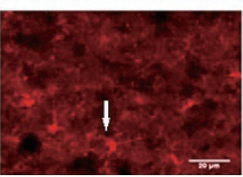

B4RT
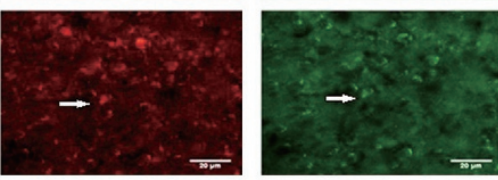

Merge
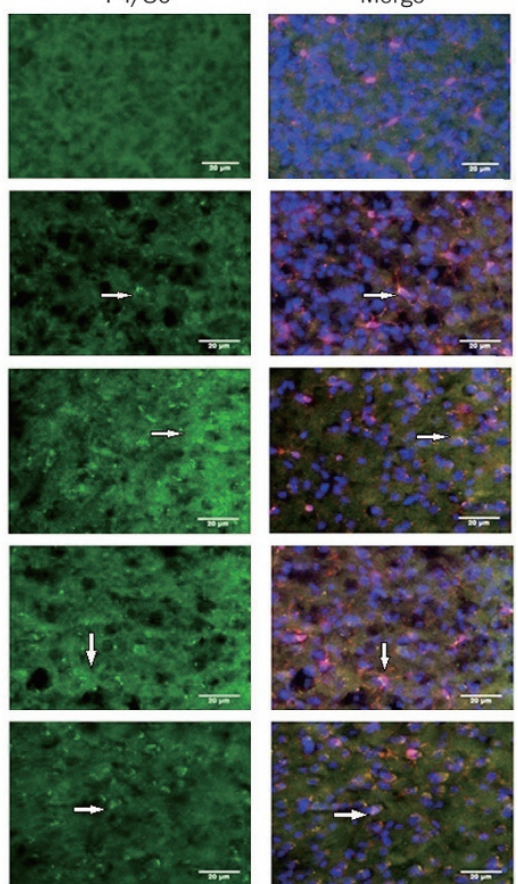

B

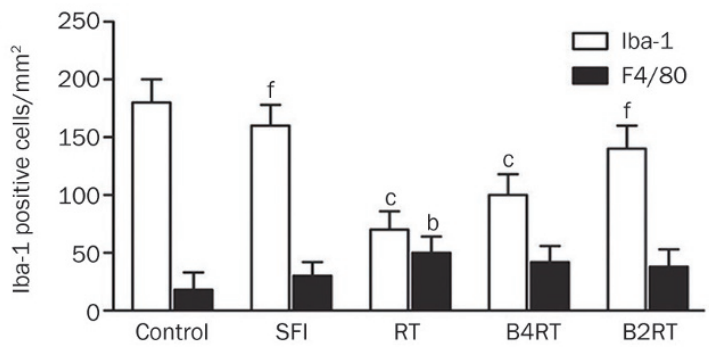

Figure 7. Phonotype changes of microglia in the brain tissues in each group. (A) Iba-1 (red) and F4/80 (green) double immunofluorescence of the mice brain tissue. The arrows indicate the cells with both lba- 1 and F4/80 positive. Scale bar=20 $\mu \mathrm{m}$. (B) The quantitative analysis of Iba-1or F4/80-positve cells. ${ }^{\mathrm{b}} P<0.05,{ }^{\mathrm{c}} P<0.01$ vs control; ${ }^{\mathrm{f}} \mathrm{P}<0.01$ vs RT-only group. $n=3$.

responses, and inhibited microglial activation in irradiationinduced brain injury. Our study with animal models showed that SFI exerted therapeutic effects on RIBI. Furthermore, we found that $20 \mathrm{~mL} \cdot \mathrm{kg}^{-1} \cdot \mathrm{d}^{-1} \mathrm{SFI}$ could reduce the expression of inflammatory cytokines in the brain tissues subjected to irradiation. Moreover, it was found that B2RT was a more effective treatment schedule. The reason why the anti-inflammatory effect in this group was more pronounced than that observed in the B4RT group may be due to the release of the pro-inflammatory cytokines after irradiation, which suggests that the SFI had a protective, anti-inflammatory role. In the B4RT group, the mice received SFI injection before, not after, irradiation. Therefore, the pre-irradiation SFI injection could not effectively develop its anti-inflammatory effect. Based on these findings, we postulated that SFI may inhibit inflammation via NF-кB pathway activation triggered by p65 phosphorylation. Our previous study ${ }^{[19]}$ showed that, SFI significantly down-

regulated the irradiation-induced expression of TNF- $\alpha$ and TGF- $\beta 1$ and maintained the level of IL-10 in mice receiving chest irradiation, which had an anti-fibrogenic effect and alleviated irradiation-induced lung injury, but the exact mechanism remains unknown.

In this study, the RIBI model was successfully established in mice after administration of 20-Gy CRT, as verified by the Morris water maze test. Mice in the B4RT and B2RT groups found the platform more easily than did the RT mice on $\mathrm{d} 4$ and 5. These data indicated that SFI treatment could alleviate the 20-Gy CRT-induced neurocognitive deficits in learning and memory. This study demonstrated that SFI treatments before or after irradiation could suppress the symptoms and prolong life span compared with the RT group.

Although the etiology of RIBI is still unknown, it is generally believed that inflammatory cytokines are one of the important pathways that lead to $\operatorname{RIBI}^{[13,41,42]}$. Therefore, a proinflammatory environment resulting from the over-expression of inflammatory mediators may be responsible for many postirradiation neuropathological complications ${ }^{[43]}$. Several previous in vivo and in vitro studies have suggested that TNF- $\alpha$ and IL-1 $\beta$ were the most important pro-inflammatory cytokines, play pivotal roles in acute and chronic inflammation, and are associated with a wide array of human diseases, including atherosclerosis, autoimmune disorders, and malignancies ${ }^{[13]}$. It was found that the expression of TNF- $\alpha$, IL-1 $\beta$ and IL- 6 in the lung tissue was biphasic: one peak was at approximately $6 \mathrm{~h}$, and the second peak was at approximately 8 weeks ${ }^{[19]}$. Our results demonstrate a complex pattern of elevated cytokines mRNA levels after cranial radiation therapy, with a clear indication of a two-phase mechanism in the molecular pathology. We observed an immediate release of pro-inflammatory cytokines at $4 \mathrm{~h}$ after irradiation. In Rube's research, the authors presumed that TNF- $\alpha$ may induce the expression of IL-1 $\beta$ and IL-6 in the irradiated brain tissue ${ }^{[44]}$, which are thought to further promote the inflammatory process. Therefore, TNF-a was thought to play a prominent role in the initiation of the cytokine cascade. Because TNF-a enhanced the production of IL- $1 \beta$ and IL-6, there was a second peak of inflammatory cytokine release, which resulted in the cascade of inflammation reactions. Our study also revealed that immediately after CRT, the brain released IL-1 $\beta$ and TNF- $\alpha$. TNF- $\alpha$, which is an inflammatory cytokine, may depend on the presence of autocrine/paracrine signals or cellular context to activate cell inflammation and proliferation, decrease survival and promote cell death ${ }^{[45]}$. IL-1 $\beta$ is produced by hematopoietic cells, such as blood monocytes, tissue macrophages, skin dendritic cells, and brain microglia, in response to stimulation. It activates complementary components, other cytokines, and IL-1 itself. Because TNF- $\alpha$ and IL-1 $\beta$ are considered the crucial mediators of inflammatory events, the irradiation-mediated overexpression of these cytokines is likely to be an important contributing factor to the pathophysiological sequelae of cranial irradiation. In this study, SFI reduced the expression of pro-inflammatory cytokines, such as TNF-a, and IL-1 $\beta$. In the present study, CRT treatment caused a sharp increase in 
A
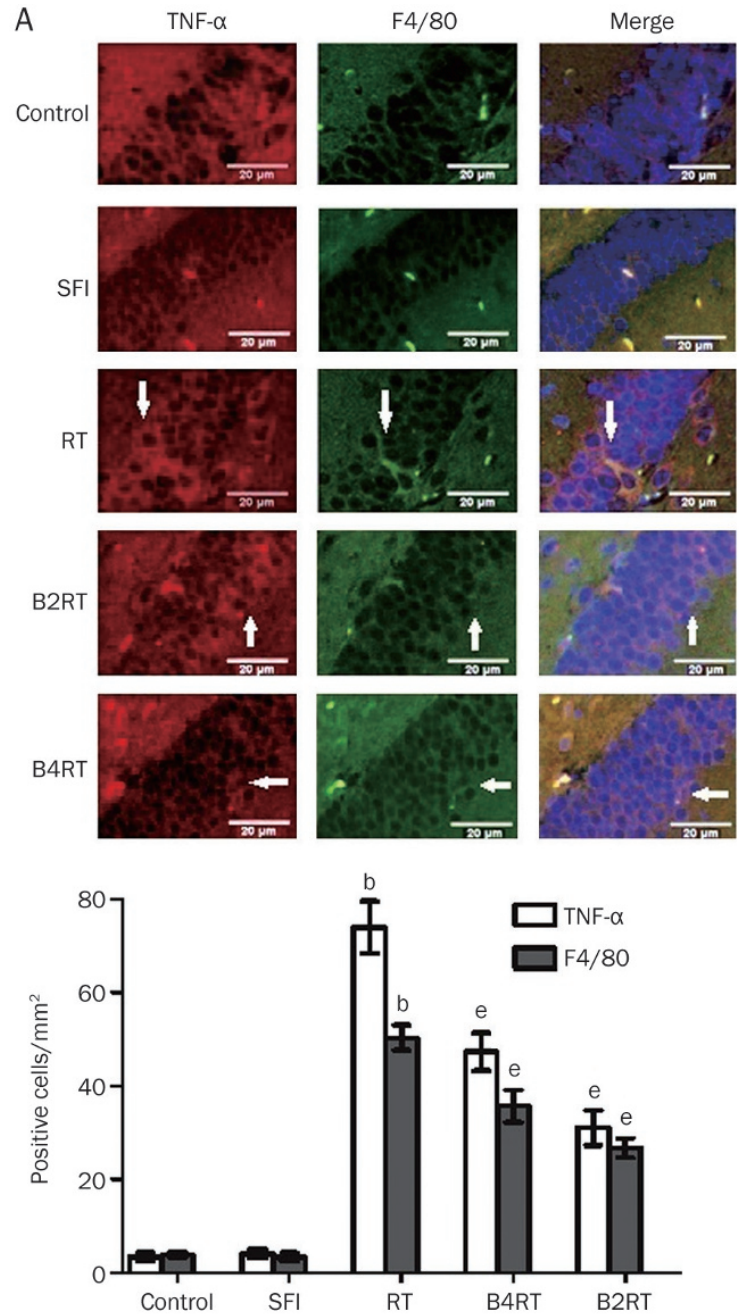

B
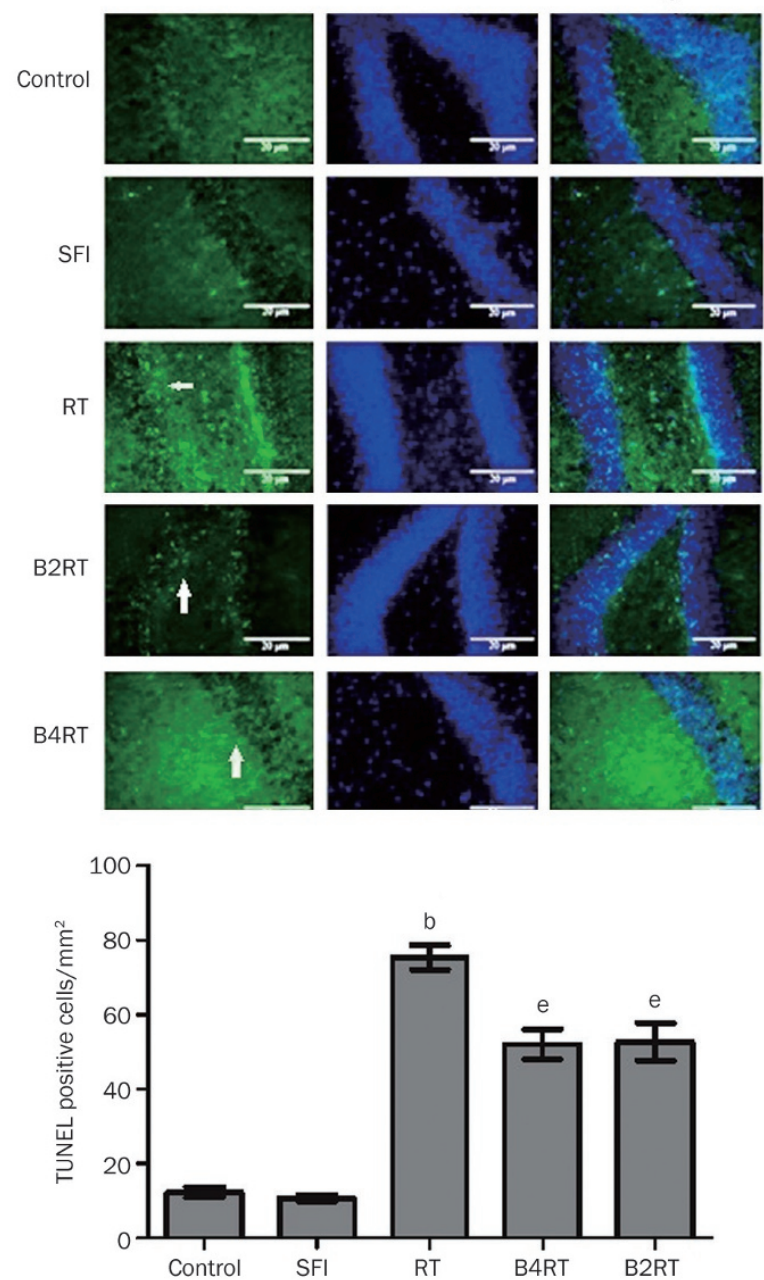

Figure 8. (A) The expression of TNF- $\alpha$ and F4/80 in CA1 of hippocampus by immunofluorescence staining $24 \mathrm{~h}$ after CRT. The arrows indicate the cells with both TNF- $\alpha$ and F4/80 positive. (B) The expression of apoptotic cells in the hippocampus by TUNEL staining. The arrows indicate the TUNELpositive cells. ${ }^{\mathrm{b}} P<0.05$ vs control; ${ }^{\mathrm{e}} P<0.05$ vs RT-only group. $n=3$. Scale bar $=20 \mu \mathrm{m}$.

the pro-inflammatory cytokines (TNF- $\alpha$ and IL-1 $\beta$ ) in murine brain. However, SFI $\left(20 \mathrm{~mL} \cdot \mathrm{kg}^{-1} \cdot \mathrm{d}^{-1}\right)$ treatment, which began two weeks before 20-Gy CRT (B2RT group), significantly reduced the production of TNF- $\alpha$ and IL- $1 \beta$, whereas no significant change was observed in the B4RT group compared with the RT-only group.

Evans blue extravasation is used to measure the vascular permeability in the CNS. Immediately after CRT, the BBB was disrupted and macrophages infiltrated into the brain. In addition, pro-inflammatory cytokines have been shown to increase BBB permeability, thus facilitating the infiltration of peripheral immune cells. The current study showed that the increased BBB permeability due to CRT was partially alleviated by the treatment with SFI. The mechanism by which SFI stabilizes the BBB may be related to its inhibitory effect on pro-inflammatory cytokine release.

NF-kB pathway activation is considered to play an important role in pro-inflammatory gene expression after stimulation $^{[46]}$. It was also reported that DNA double strands breaks
(DSBs) may activate the NF-kB pathway after irradiation ${ }^{[47]}$. In our report, the level of the phosphorylated p65 protein was upregulated after CRT, and SFI inhibited this upregulation.

PIDD was originally identified as a gene that exhibits p53-dependent expression, and it may act to promote p53induced cell death. In addition, PIDD was believed to mediate the DNA damage-induced NF-kB activation ${ }^{[48,49]}$. PIDD expression could upregulate the production of genotoxicstress-induced NF- $\mathrm{KB}$ by augmenting the sumoylation and ubiquitination of NF-KB essential modulator (NEMO ${ }^{[48]}$. PIDD-FL is constitutively cleaved into three fragments by autoproteolysis: an N-terminal fragment (PIDD-N) and a C-terminal fragment (PIDD-C), which is further cleaved into PIDD-CC. Similar to previous research ${ }^{[49]}$, our study detected only a negligible expression of PIDD-FL. PIDD-C binds to the receptor interacting protein (RIP-1) and NEMO, which controls the pro-survival NF-kB pathway, whereas PIDD-CC, together with RIP-associated Ich-1/CED homologous protein with death domain (RAIDD), can induce apoptosis. This 
study showed that PIDD-C reached a peak at $3 \mathrm{~h}$ after irradiation in the brain. In contrast to PIDD-C, PIDD-CC is thought to be pro-apoptotic. A previous study revealed that PIDD-CC was upregulated in the brain after irradiation ${ }^{[50-53]}$. Similarly, our study showed that PIDD-CC was significantly increased at $3 \mathrm{~h}$ after irradiation, which indicates that the roles of different PIDD segments differ in cell survival and apoptosis after irradiation, and NF-kB pathway activation precedes apoptosis. PIDD-C-induced neuro-inflammation and apoptosis were both implicated in irradiation-induced NF-KB pathway activation, and PIDD-CC was involved in radiation-induced apoptosis and played an important role in RIBI.

\section{Conclusion}

This study demonstrated that SFI treatment could attenuate the structural and biochemical abnormalities in a mouse model of irradiation-induced acute brain injury by inhibiting NF-kB-mediated expression of inflammatory genes. SFI promises to be a useful alternative for the prevention of irradiationinduced brain injury.

\section{Acknowledgements}

This work was supported by grants from the National Natural Science Foundation of China (81573090 and 81172595), Postdoctor Foundation of China (20100480905), Post-doctor Special Foundation of China (201104440), and China Oncology Clinical Science Fund (Y-L2011-002).

\section{Author contribution}

Jian ZHANG, Gang WU and Xiao-rong DONG designed research; Jian ZHANG, Fan TONG and Ji-hua DONG performed research; Fan TONG contributed new reagents and analytical tools; Qian CAI and Ling-juan CHEN analyzed data; Jian ZHANG, Fan TONG and Xiao-rong DONG wrote the paper.

\section{References}

1 Khuntia D, Brown P, Li J and Mehta MP. Whole-brain radiotherapy in the management of brain metastasis. J Clin Oncol 2006; 24: 1295304.

2 Johannesen TB, Langmark F and Lote K. Cause of death and longterm survival in patients with neuro-epithelial brain tumours: a population-based study. Eur J Cancer 2003; 39: 2355-63.

3 Cole AM, Scherwath A, Ernst G, Lanfermann H, Bremer M and Steinmann D. Self-reported cognitive outcomes in patients with brain metastases before and after radiation therapy. Int J Radiat Oncol Biol Phys 2013; 87: 705-12.

4 Beltran C, Naik M and Merchant TE. Dosimetric effect of target expansion and setup uncertainty during radiation therapy in pediatric craniopharyngioma. Radiother Oncol 2010; 97: 399-403.

5 Li J, Bentzen SM, Li J, Renschler M and Mehta MP. Relationship between neurocognitive function and quality of life after whole-brain radiotherapy in patients with brain metastasis. Int J Radiat Oncol Biol Phys 2008; 71: 64-70.

6 Welzel G, Fleckenstein K, Mai SK, Hermann B, Kraus-Tiefenbacher U and Wenz $F$. Acute neurocognitive impairment during cranial radiation therapy in patients with intracranial tumors. Strahlenther Onkol
2008; 184: 647-54.

7 Welzel G, Fleckenstein K, Schaefer J, Hermann B, Kraus-Tiefenbacher $\mathrm{U}$, Mai SK, et al. Memory function before and after whole brain radiotherapy in patients with and without brain metastases. Int J Radiat Oncol Biol Phys 2008; 72: 1311-8.

8 Schnegg $\mathrm{Cl}$, Kooshki M, Hsu FC, Sui G and Robbins ME. PPARס prevents radiation-induced proinflammatory responses in microglia via transrepression of NF-kappaB and inhibition of the PKC $\alpha / M E K 1 / 2 /$ ERK1/2/AP-1 pathway. Free Radic Biol Med 2012; 52: 1734-43.

9 Liu JL, Tian DS, Li ZW, Qu WS, Zhan Y, Xie MJ, et al. Tamoxifen alleviates irradiation-induced brain injury by attenuating microglial inflammatory response in vitro and in vivo. Brain Res 2010; 1316: 101-11.

10 Zhao W and Robbins ME. Inflammation and chronic oxidative stress in radiation-induced late normal tissue injury: therapeutic implications. Curr Med Chem 2009; 16: 130-43.

11 Xue J, Dong JH, Huang GD, Qu XF, Wu G, Dong XR. NF-kB signaling modulates radiation-induced microglial activation. Oncol Rep 2014; 31: 2555-60.

12 Chiang CS, Hong JH, Stalder A, Sun JR, Withers HR and McBride WH. Delayed molecular responses to brain irradiation. Int J Radiat Biol 1997; 72: 45-53.

13 Lee WH, Sonntag WE, Mitschelen M, Yan H and Lee YW. Irradiation induces regionally specific alterations in pro-inflammatory environments in rat brain. Int J Radiat Biol 2010; 86: 132-44.

14 Stoll $G$ and Jander S. The role of microglia and macrophages in the pathophysiology of the CNS. Prog Neurobiol 1999; 58: 233-47.

15 Gebicke-Haerter PJ. Microglia in neurodegeneration: molecular aspects. Microsc Res Tech 2001; 54: 47-58.

16 Pocock JM and Liddle AC. Microglial signalling cascades in neurodegenerative disease. Prog Brain Res 2001; 132: 555-65.

17 Luo XG, Chen SD. The changing phenotype of microglia from homeostasis to disease. Transl Neurodegener 2012; 1: 9.

18 Ramanan S, Zhao W, Riddle DR and Robbins ME. Role of PPARs in radiation-induced brain injury. PPAR Res 2010; 2010: 234975.

19 Abbott NJ. Dynamics of CNS barriers: evolution, differentiation, and modulation. Cell Mol Neurobiol 2005; 25: 5-23.

20 Misra A, Ganesh S, Shahiwala A and Shah SP. Drug delivery to the central nervous system: a review. J Pharm Pharm Sci 2003; 6: 25273.

21 Chodobski A, Zink BJ and Szmydynger-Chodobska J. Blood-brain barrier pathophysiology in traumatic brain injury. Transl Stroke Res 2011; 2: 492-516.

22 Ganss R, Ryschich E, Klar E, Arnold B and Hammerling GJ. Combination of T-cell therapy and trigger of inflammation induces remodeling of the vasculature and tumor eradication. Cancer Res 2002; 62: 1462-70.

23 Dong J, Su SY, Wang MY, Zhan Z. Shenqi fuzheng, an injection concocted from Chinese medicinal herbs, combined with platinum-based chemotherapy for advanced non-small cell lung cancer: a systematic review. J Exp Clin Cancer Res 2010; 29: 137.

24 Wang J, Tong X, Li P, Cao H and Su W. Immuno-enhancement effects of Shenqi Fuzheng Injection on cyclophosphamide-induced immunosuppression in Balb/c mice. J Ethnopharmacology 2012; 139: 788-95.

25 Dong XR, Wang JN, Liu L, Chen X, Chen MS, Chen J, et al. Modulation of radiation-induced tumour necrosis factor-alpha and transforming growth factor beta1 expression in the lung tissue by Shengqi Fuzheng injection. Mol Med Rep 2010; 3: 621-7.

26 Wang J, Tong X, Li P, Liu M, Peng W, Cao H, et al. Bioactive components on immuno-enhancement effects in the traditional 
Chinese medicine Shenqi Fuzheng Injection based on relevance analysis between chemical HPLC fingerprints and in vivo biological effects. J Ethnopharmacol 2014; 155: 405-15.

27 Ma XQ, Shi Q, Duan JA, Dong TTX and Tsim KWK. Chemical analysis of Radix Astragali (Huangqi) in China: a comparison with its adulterants and seasonal variations. J Agric Food Chem 2002; 50: 4861-6.

28 Li CY, Xu HX, Han QB and Wu TS. Quality assessment of Radix Codonopsis by quantitative nuclear magnetic resonance. J Chromatogr A 2009; 13: 2124-29.

29 Hodges H, Katzung N, Sowinski P, Hopewell JW, Wilkinson $\mathrm{JH}$, Bywaters T, Rezvani M. Late behavioural and neuropathological effects of local brain irradiation in the rat. Behav Brain Res 1998; 91 : 99-114.

30 Pezner RD, Lipsett JA, Archambeau JO, Fine RM and Moss WT. Highdose fractionated radiation therapy for select patients with brain metastases. Radiology 1981; 140: 479-81.

31 Cevik NG, Orhan N, Yilmaz CU, Arican N, Ahishali B, Kucuk M, et al. The effects of hyperbaric air and hyperbaric oxygen on blood-brain barrier integrity in rats. Brain Res 2013; 1531: 113-21.

32 Overbergh L, Giulietti A, Valckx D, Decallonne R, Bouillon R and Mathieu C. The use of real-time reverse transcriptase PCR for the quantification of cytokine gene expression. J Biomol Tech 2003; 14: 33-43.

33 Acharya MM, Christie LA, Lan ML, Donovan PJ, Cotman CW, Fike JR, et al. Rescue of radiation-induced cognitive impairment through cranial transplantation of human embryonic stem cells. Proc Natl Acad Sci U S A. 2009; 106: 19150-5.

34 Pan L. Practical road of "the numeral turn Chinese herbal medicine" for Shenqi fuzheng injection. J China Prescript Drug (Chin) 2009; 1: 37-9.

35 Zhong, ZH (2009) Dictate history: a national new medicine that come from experiential prescription. J China Prescript Drug (Chin) 2009; 1: 33-6.

36 Shan BE, Yoshida Y, Sugiura T, Yamashita U. Stimulating activity of Chinese medicinal herbs on human lymphocytes in vitro. Int $\mathrm{J}$ Immunopharmacol 1999; 21: 149-59.

37 Yuan Y, Sun M, Li KS. Astragalus mongholicus polysaccharide inhibits lipopolysaccharide-induced production of TNF-alpha and interleukin-8. World J Gastroenterol 2009; 15: 3676-80.

38 Dong X, Luo M, Huang G, Zhang J, Tong F, Cheng Y, et al. Relationship between irradiation-induced neuro-inflammatory environments and impaired cognitive function in the developing brain of mice. Int J Radiat Biol 2015; 91: 224-39.

39 Jung IH, Jang SE, Joh EH, Chung J, Han MJ, Kim DH. Lancemaside A isolated from Codonopsis lanceolata and its metabolite echinocystic acid ameliorate scopolamine-induced memory and learning deficits in mice. Phytomedicine 2012; 20: 84-8.

40 Lee JS, Kim HG, Han JM, Kim DW, Yi MH, Son SW, et al. Ethanol extract of Astragali Radix and Salviae Miltiorrhizae Radix, Myelophil, exerts anti-amnesic effect in a mouse model of scopolamine-induced memory deficits. J Ethnopharmacol 2014; 153: 782-92.

41 Lee YW, Cho HJ, Lee WH and Sonntag WE. Whole brain radiationinduced cognitive impairment: pathophysiological mechanisms and therapeutic targets. Biomol Ther (Seoul) 2012; 20: 357-70.

42 Greene-Schloesser D and Robbins ME. Radiation-induced cognitive impairment-from bench to bedside. Neuro Oncol 2012; 14: iv37-44.

43 Hsieh HL and Yang CM. Role of redox signaling in neuroinflammation and neurodegenerative diseases. Biomed Res Int 2013; 2013: 484613.

44 Rübe CE, Wilfert F, Palm J, König J, Burdak-Rothkamm S, Liu L, et al. Irradiation induces a biphasic expression of pro-inflammatory cytokines in the lung. Strahlenther Onkol 2004; 180: 442-8.

45 Ardestani S, Deskins DL and Young PP. Membrane TNF-alphaactivated programmed necrosis is mediated by Ceramide-induced reactive oxygen species. J Mol Signal 2013; 8: 12.

46 Lawrence T. The nuclear factor NF-kappaB pathway in inflammation. Cold Spring Harb Perspect Biol 2009; 1: a001651.

47 Miyamoto S: Nuclear initiated NF-kappaB signaling: NEMO and ATM take center stage. Cell Res 2011; 21: 116-30.

48 Yang Y, Xia F, Hermance N, Mabb A, Simonson S, Morrissey S, Gandhi P, et al. A cytosolic ATM/NEMO/RIP1 complex recruits TAK1 to mediate the NF-kappaB and p38 mitogen-activated protein kinase (MAPK)/ MAPK-activated protein 2 responses to DNA damage. Molecular and Cellular Biology 2011; 31: 2774-86.

49 Janssens S, Tinel A, Lippens S and Tschopp J. PIDD mediates NFkappaB activation in response to DNA damage. Cell 2005; 123 : 1079-92.

50 Niizuma K, Endo H, Nito C, Myer DJ, Kim GS and Chan PH. The PIDDosome mediates delayed death of hippocampal CA1 neurons after transient global cerebral ischemia in rats. Proc Natl Acad Sci USA 2008; 105: 16368-73.

51 McCoy F, Eckard L and Nutt LK. Janus-faced PIDD: a sensor for DNA damage-induced cell death or survival? Mol Cell 2012; 47: 667-8.

52 Pick R, Badura S, Bösser S and Zörnig M. Upon intracellular processing, the $\mathrm{C}$-terminal death domain-containing fragment of the p53-inducible PIDD/LRDD protein translocates to the nucleoli and interacts with nucleolin. Biochem Biophys Res Commun 2006; 349: 1329-38.

53 Logette E, Schuepbach-Mallepell S, Eckert MJ, et al. PIDD orchestrates translesion DNA synthesis in response to UV irradiation. Cell Death Diffe 2011; 18: 1036-45. 